\title{
OPCIONES HURLO: UNA CLASE DE DERIVADO METEOROLÓGICO PARA LA PROTECCIÓN FRENTE AL RIESGO DE HURACANES*
}

\section{HURLO OPTIONS: A WEATHER DERIVATIVE FOR HURRICANE RISK MANAGEMENT}

\author{
MARÍA JOSÉ PÉREZ-FRUCTUOSO** \\ Fecha de recepción: 27 de abril de 2021 \\ Fecha de aceptación:30 de mayo de 2021 \\ Disponible en línea: 30 de junio de 2021
}

Para citar este artículo/To cite this article

Perez-Fructuoso, María José, Opciones HuRLO: una clase de derivado meteorológico para

la protección frente al riesgo de huracanes, 54 Rev.Ibero-Latinoam.Seguros, 275-286 (2021).

https://doi.org/10.11144/Javeriana.ris54.ohcd

doi:10.11144/Javeriana.ris54.ohcd

\footnotetext{
"Artículo de reflexión

** Doctora Europea en Economía, Doctora en Ciencias Económicas y Empresariales, Licenciada en Ciencias Actuariales y Financieras. Licenciada en Ciencias Económicas y Empresariales Profesora Titular del Área de Economía Financiera y Contabilidad. Madrid Open University (UDIMA). ORCID: 0000-00023252-1631 Contacto: mariajose.perez@udima.es
} 


\section{RESUMEN}

Este artículo analiza las características, el funcionamiento y la estructura de un nuevo derivado meteorológico definido como una opción sobre commodities que protege contra el riesgo de que una determinada zona costera de los EE.UU. se vea afectada por la ocurrencia de un huracán durante un año determinado.

Palabras clave: derivados meteorológicos, riesgo de huracanes, gestión del riesgo, pari-mutuel

\section{ABSTRACT}

This article analyzes the characteristics, operation and structure of a new weather derivative defined as a commodity option that protects against the risk that a certain coastal area of the United States will be affected by the occurrence of a hurricane during a given year.

Key Words: weather derivatives, hurricane risk, risk management, pari-mutuel

\section{SUMARIO}

1. Introducción 2. ¿Qué son las opciones HuRLO? 3. Descripción y funcionamiento del mercado de las opciones HuRLO 3.1. Descripción del mercado 3.2. Participantes en el mercado 3.3. Características de la negociación 4. Cálculo del precio de las opciones HuRLO 5. Conclusiones Referencias bibliográficas 


\section{INTRODUCCIÓN}

El gran aumento de las pérdidas aseguradas derivadas de la ocurrencia de catástrofes naturales ha provocado una insuficiencia de la cobertura tradicional del reaseguro motivando la búsqueda de nuevas formas alternativas de transferencia del riesgo a través de los mercados de capital. Los huracanes se encuentran entre los eventos naturales más catastróficos. Si bien es cierto que el número de huracanes que tocan tierra en EE.UU. permanece estable desde 1900 entorno a una media de 18 huracanes por década, sus consecuencias financieras pueden ser devastadoras. Actualmente se estima que la ocurrencia de un huracán puede generar más de 500.000 millones de dólares en pérdidas aseguradas más incluso que las registradas por el huracán Katrina.

Existen seguros que protegen contra los daños provocados por la ocurrencia de un huracán, a propietarios de viviendas o por interrupción de la actividad comercial, especialmente donde la ocurrencia de este fenómeno es más importante esto es el sureste de Estados Unidos, alrededor del Golfo de México y en el oeste del Pacífico Norte. Estos seguros normalmente se contratan con una franquicia muy elevada, que supone entre $2 \%$ y el $15 \%$ del valor de la vivienda o del lugar de trabajo, y no suelen cubrir las zonas exteriores de una casa o negocio (incluidos los jardines, la iluminación exterior, los muelles y las vallas). Por su parte, el seguro de interrupción de la actividad empresarial no suele estar disponible y cuando lo está, es muy costoso y con duración y alcance limitados (Weather Risk Solutions, 2010a).

La concentración y el crecimiento de la población, y por tanto de valores materiales asegurados, en zonas urbanas propensas, o potencialmente propensas, a este tipo de desastres tiene como consecuencia que el mercado asegurador mundial tenga una mayor exposición a pérdidas catastróficas que puede comprometer la solvencia y la estabilidad de las compañías que operan en él, si no son capaces de obtener la financiación y diversificación necesarias para asegurar su viabilidad futura y la protección de sus asegurados. Para complementar el mercado de seguros tradicional en los años 90 y principios de los 2000 surgieron instrumentos financieros denominados Insurance Linked Securities cuyo objetivo es incrementar las posibilidades en el mercado asegurador y, por tanto, la capacidad de suscripción, ofertando mayores coberturas en aquellos casos en los que las coberturas existentes hasta el momento han sido insuficientes o nulas. (Cummins et al. 2013). Estos instrumentos conocidos como opciones, bonos catastróficos y swaps entre otros permiten a las aseguradoras trasladar parte de su riesgo a los inversores quienes toman posiciones en la ocurrencia y el coste de las catástrofes (Pérez-Fructuoso, 2005). Además, según estén diseñados, el riesgo moral y el riesgo de crédito pueden ser muy bajos, aunque como contrapartida siempre se produce un aumento del riesgo de base.

En el año 2008 Weather Risk Solutions (WRS) lanzó al mercado un nuevo instrumento denominado Hurricane Risk Landfall Option, opciones HuRLO, cuyo objetivo es ayudar a los inversores a gestionar el riesgo financiero ante la ocurrencia de catástrofes tomando posiciones sobre las apuestas que realizan de forma conjunta acerca de la primera llegada a tierra de huracanes (pari-mutuel first-by-the-post) (Boyer et al., 2020). Estas opciones son contratos derivados meteorológicos estandarizados, 
negociados en un mercado organizado, que facilitan el traslado del riesgo financiero de que un huracán toque tierra por primera vez en el condado o región en el que vive un asegurado a todos aquellos que seleccionan una zona geográfica cubierta diferente a cambio de un precio. En caso de que el asegurado esté ubicado en la zona geográfica en la que el huracán toca tierra por primera vez y los daños económicos provocados por el mismo se estima que alcanzarán o superarán el umbral de daños de 1 millón de dólares, y haya adquirido una HuRLO para esa zona, recibirá un importe de liquidación procedente de los fondos pagados por todos los que seleccionaron zonas diferentes, es decir, reciben una parte del fondo de riesgo mutualizado. Así pues, las opciones HuRLO se autofinancian completamente y no requieren subvenciones de los contribuyentes.

Diversos autores han abordado la descripción y análisis de estos nuevos instrumentos derivados. WiLKs, et al. (2011) y (2014) describen, desde un punto de vista tanto teórico como empírico, un método para la gestión del riesgo de huracanes a partir de las opciones HuRLO. Boyer et al. (2020) analizan el funcionamiento del mercado de las HuRLO y presenta una propuesta de modelización de las decisiones que se toman en dicho mercado por los operadores adversos al riesgo que quieren cubrirse contra pérdidas catastróficas derivadas de la ocurrencia de un huracán. OU-YANG (2010) y OU-YANG et al. (2011) analizan definen las opciones HuRLO como una apuesta pari-mutuel y determinan si las operaciones con estas opciones pueden proteger a los inversores con aversión al riesgo contra pérdidas catastróficas.

En este trabajo se realiza un análisis de las opciones HuRLO como nuevo instrumento de cobertura de las pérdidas económicas asociadas a la ocurrencia de huracanes, así como de transferencia de dicho riesgo a los mercados de capital.

Tras esta introducción, en la sección 2 se definen las opciones HuRLO y sus principales características. Seguidamente, la sección 3 se destinan a describir de manera exhaustiva el funcionamiento del mercado en el que estas opciones se negocian, los participantes que en él intervienen y las características fundamentales de su negociación. La sección 4 presenta una forma de calcular el precio de este tipo de opciones en el mercado, considerándolas como una apuesta pari-mutuel. Y finalmente, la sección 5 concluye.

\section{2. ¿QUÉ SON LAS OPCIONES HURLO?}

Los contratos sobre huracanes que se analizan en este artículo se conocen con el nombre de Hurricane Risk Landfall Option, opciones HuRLO u opciones cuyo subyacente es el riesgo de que un huracán toque tierra por primera vez, y se definen legalmente como opciones binarias sobre commodities según la Commodity Exchange Act (Ley de Intercambio de Materias Primas).

Estas opciones son instrumentos financieros derivados cuyo objetivo es proporcionar una forma simple y eficiente, en términos de coste, de protegerse o especular con el riesgo de que una determinada región costera o condado de EE.UU. sea la primera zona geográfica en sufrir las consecuencias económicas de la ocurrencia de un huracán. Las 
zonas geográficas consideradas en estos contratos son el Golfo de México y las costas del este de EE.UU. entre las fronteras de México y Canadá.

Se estructuran como opciones de compra (call options) y el subyacente se define de forma paramétrica en función de la ocurrencia o no, del lugar en el que un huracán tocará tierra por primera vez en un año natural y de si dicho huracán provocará un determinado umbral de pérdidas económicas también denominado desencadenante monetario (Weather Risk Solutions, 2010c). Este desencadenante es la cantidad mínima de pérdidas económicas totales que se estima que ha causado un huracán que toca tierra en primer lugar en una de las zonas geográficas especificada en el contrato de opción. Dicha estimación la realiza Eqecat, $\operatorname{Inc}^{1}$., y es necesaria para que las opciones HuRLO de una determinada zona geográfica en la que se considera que el huracán toca tierra por primera vez, sean ejercitadas y liquidadas automáticamente.

Más concretamente, el subyacente de estas opciones puede definirse de tres formas (Weather Risk Solutions, 2010b):

- que un huracán toque tierra en primer lugar en uno (y solo uno) de los 74 condados o regiones de la costa Atlántica o del Golfo de los EE.UU. y que los daños económicos estimados en esa zona alcancen o superen 1 millón de dólares en pérdidas aseguradas;

- o que ningún huracán toque tierra;

- o que se estime que el o los huracanes que tocan tierra por primera vez causan menos de 1 millón de dólares de daños en pérdidas totales en la zona afectada en primer lugar.

La opción HuRLO se ejerce automáticamente y da derecho a percibir un importe de liquidación si alguno de los tres sucesos comentados anteriormente se produce antes del 15 de diciembre del año natural cubierto, momento en el que vence la opción. Son por tanto opciones de vencimiento europeo. Y su riesgo es limitado e identificable, esto es, el comprador de este tipo opciones limita su pérdida al importe pagado por la opción.

Se negocian en series. En el lanzamiento inicial de estas opciones se ofrecieron solo dos series: una para cubrir los daños producidos por la primera llegada a tierra de un huracán en una zona geográfica determinada, y otra para la llegada a tierra de un segundo huracán. Actualmente, cada año natural se emiten tres series, aunque Wather Risk Solutions puede emitir más series si lo considera necesario. La liquidación de las series se realiza a partir del análisis de los datos anuales de huracanes que tocan tierra en EE.UU. ocurridos desde 1880 que proceden de dos fuentes, el centro Nacional de Huracanes (CNH) y la Oficina del Censo de EE.UU. Dicho análisis establece que el $90 \%$ de todos los años analizados, tocan tierra en la costa de EE.UU. tres o menos huracanes. Basándonos en estos datos, la serie 1 de las HuRLO liquida cuando se produce el primer huracán que toca tierra en EE.UU. y causa al menos un millón de

1 Eqecat, Inc es una empresa de la modelización de riesgos catastróficos que ofrece productos y servicios de última generación a los mercados mundiales de seguros de daños y responsabilidad civil, reaseguros y mercados financieros. 
dólares en pérdidas totales. La serie 2 liquida cuando se produce el segundo huracán que causa al menos un millón de dólares de pérdidas totales. Y así sucesivamente. Por ejemplo, en los años en los que solo toca tierra un huracán en EE.UU., los compradores de los contratos de la serie 2 (y superiores) reciben un pago, pero los compradores de la serie 1 no. En el caso de que ningún huracán toque tierra en un año natural (o si ningún segundo huracán toca tierra o se estima que los huracanes que tocan tierra causan menos de 1 millón de dólares de pérdidas totales en la zona afectada), las opciones de la serie asociada a la cobertura del riesgo de que el/los huracanes no toquen tierra (HuRLO No Landfall) se ejercen automáticamente y sus titulares reciben el importe de liquidación (Weather Risk Solutions, 2010b).

Cuando se emiten tres series de cada opción HuRLO, pueden darse 75 resultados diferentes en cada serie para un total de 225 que se pueden comprar. Estos resultados incluyen cada uno de los 74 condados o regiones costeras de Estados Unidos, así como el resultado de la no llegada a tierra de huracanes.

Si se produce un huracán, los titulares de la opción ganadora, que son los poseedores de opciones para el lugar en el que el huracán ha tocado tierra por primera vez, reciben un pago y, por tanto, la opción vence in-the-money, mientras que los titulares de todas las demás opciones no reciben nada y dichas opciones vencen out-of-the-money. Al final de la temporada, los titulares de la opción que cubre la no llegada a tierra de huracanes de todas las series que aún no se han materializado reciben un pago, mientras que todas las demás opciones carecen de valor. Si un huracán golpea dos zonas geográficas cubiertas, se considera como un segundo huracán si los puntos de contacto están separados por más de 150 millas náuticas.

\section{DESCRIPCIÓN Y FUNCIONAMIENTO DEL MERCADO DE LAS OPCIONES HURLO}

\subsection{Descripción del mercado}

La estructura del mercado de opciones HuRLO difiere de la de los mercados tradicionales de derivados meteorológicos. Las primeras HuRLO se lanzaron al mercado en octubre de 2008 a través una Plataforma Electrónica de Negociación de Weather Risk Solutions a la que se accede directamente a través de internet. Esta plataforma era un sistema propio de entrada y ejecución de órdenes operado por Weather Risk Solutions y utilizado por el mercado para la colocación y ejecución de órdenes o la recogida y transmisión de información relativa a estas opciones (Weather Risk Soluctions, 2010d). Desde 2009, se negocian en EXBOT, tablero de negociación sujeto a la jurisdicción legal exclusiva de la Comisión de Comercio de Futuros de Materias Primas de Estados Unidos (U.S. Commodity Futures Trading Commission) y en el que solo pueden participar inversores institucionales tales como bancos, compañías de seguros, fondos de cobertura, grandes empresas comerciales y ciertos individuos con elevada riqueza. 
Existe un mercado primario de opciones HuRLO y un mercado secundario (Weather Risk Solutions, 2010c). El primero de ellos se define como el mercado en el que los participantes cualificados pueden comprar HuRLO en cada uno de los setenta y cinco resultados diferentes de una serie de opciones HuRLO determinada. El mercado secundario es aquel en el que los participantes cualificados que poseen HuRLOs pueden venderlas a otros participantes cualificados. Este mercado secundario ofrece, pues, un mecanismo para que los participantes que tienen posiciones las transfieran a otros participantes con creencias diferentes, a precios acordados. Es importante tener en cuenta que la compra de opciones HuRLO en el mercado secundario no aumenta el número de HuRLOs en circulación a efectos de liquidación y ejercicio automático. Los vendedores en este mercado deben ser propietarios de la opción HuRLO que intentan vender y no se permite la venta al descubierto en ninguno de los dos mercados.

La negociación, tanto en el mercado primario como en el secundario, o en ambos, de una o más series de opciones HuRLO puede ser suspendida en cualquier momento por razones meteorológicas u operativas si así lo determina Weather Risk Solutions.

En todo caso, la negociación en el mercado primario de la serie de menor numeración debe suspenderse inmediatamente después de que el Centro Nacional de Huracanes $(\mathrm{CNH})$ advierta de la posible ocurrencia de un huracán en cualquier lugar de la costa atlántica de los EE.UU. Esta suspensión se mantendrá hasta que sea posible determinar si el huracán del que ha alertado el $\mathrm{CNH}$ ha tocado tierra con la fuerza de un huracán y si se estima que ha causado al menos 1.000.000 de dólares en daños económicos en la primera zona en la que ha tocado tierra. Después de cualquier suspensión, la negociación puede reanudarse si Weather Risk Solutions concluye que ningún huracán ha tocado tierra, que cualquier tormenta que hay tocado tierra tierra lo ha hecho con una fuerza inferior a la de un huracán, o que no se ha alcanzado el umbral de daños establecido.

La negociación de una serie concreta de las opciones HuRLO, salvo en el caso de aquellas que cubren la no llegada a tierra de huracanes, finaliza en la fecha más temprana de entre las siguientes:

1) 2:00 p.m. del 15 de diciembre, o

2) cuando Weather Risk Solutions decida finalizarla tras una suspensión por él mismo establecida, 0

3) cuando Weather Risk Solutions determine:

i. que un Huracán ha tocado tierra en la Costa de los Estados Unidos;

ii. que el primer lugar de llegada a tierra es uno de los lugares de llegada de huracanes recogidos en las especificaciones del contrato y

iii. que se ha alcanzado el umbral de daños establecido.

Para las opciones HuRLO que cubren la no llegada a tierra de huracanes, la negociación en el mercado primario de la serie 1 finaliza a las 2:00 p.m. del 15 de octubre del año de negociación, siempre y cuando dicha negociación no haya terminado antes y por tanto la serie 1 no se haya liquidado ya. La negociación de estas opciones para la serie 2 terminará a las 2:00 p.m. del 1 de octubre del año de negociación, siempre que la 
negociación de la serie 1 o de la serie 2 no ya haya terminado y por tanto la serie 1 o la serie 2 todavía no se hayan liquidado. Las opciones HuRLO que cubren la no llegada a tierra de huracanes de la serie 3 dejan de negociarse a las 2:00 p.m. del 9 de septiembre del año de negociación, a menos que la negociación de las series 1, 2 o 3 ya haya terminado y por tanto se hayan liquidado. Si un huracán toca tierra en la costa de EE.UU. antes del 9 de septiembre (y se cumple el umbral de daños), Weather Risk Solutions puede ampliar la fecha de finalización de la negociación en el mercado primario de las series 2 y 3 de las opciones HuRLO que cubren la no llegada a tierra de huracanes hasta el 15 de octubre y el 1 de octubre, respectivamente. Si Weather Risk Solutions decide ampliar las series en negociación, deberá notificar a través de la plataforma la última fecha de negociación en el mercado primario para las opciones HuRLO que cubren la no ocurrencia de huracanes (Weather Risk Soluctions, 2010a y 2010c).

En cualquier caso, Weather Risk Solutions y el mercado de negociación determinan de mutuo acuerdo para cualquier HuRLO y/o serie de HuRLO cuándo, como consecuencia de requisitos legales, condiciones de mercado u otro tipo de emergencia, es necesario interrumpir la negociación, retrasar la apertura o suspender nuevamente la negociación, y cuándo debe reanudarse o abrirse la negociación.

\subsection{Participantes en el mercado}

En cuanto a los participantes en este tipo de mercado, las opciones HuRLO ofrecen un mecanismo sencillo para que los aseguradores puedan cubrirse contra el riesgo de huracanes ya que es posible comprarlas en cualquier momento sin tener que encontrar a otro participante que quiera figurar de contraparte de la operación vendiendo este tipo de opciones a un precio determinado. Cada participante en el mercado puede adquirir un número ilimitado de opciones HuRLO en cualquier resultado o combinación de ellos y están disponibles en cualquier momento hasta que finalice la negociación de la serie HuRLO en cuestión. Pero los aseguradores no son los únicos que pueden comprar las opciones HuRLO. Estos instrumentos también son utilizados por aquellos inversores que quieren especular sobre el lugar en el que tocará tierra por primera vez una tormenta que posteriormente se convierte en huracán aportando así liquidez para financiar las posiciones de los aseguradores, de igual forma que muchos mercados financieros.

Todos los participantes en el mercado tienen acceso a la misma información a saber: número de opciones compradas para cada resultado de las opciones; importe de todas las primas de las opciones pagadas hasta la fecha en cada serie; precio de cada resultado de las opciones, probabilidad de que un huracán toque tierra por primera vez en cada uno de los condados o regiones considerados y la probabilidad de no ocurrencia de un huracán; y el importe de liquidación si el huracán toca tierra por primera vez en la ubicación cubierta por la HuRLO y se cumple el umbral de daños (o en el caso de la opción que cubre la no ocurrencia de un huracán, el importe de liquidación si ningún huracán toca tierra o no se cumple el umbral de daños para los huracanes que tocan tierra) (Weather Risk Soluctions, 2010a y 2010c).

Es importante notar que la mayor parte de dichos participantes no tienen experiencia en predecir si los huracanes tocarán tierra y dónde, pero todos ellos tienen acceso 
a las decisiones de compra tomadas por otros inversores que, deberían reflejar de forma racional las previsiones objetivas realizadas por el CNH. Sin embargo, sigue existiendo la posibilidad de que se produzcan distorsiones en el mercado si los participantes creen que poseen información privilegiada sobre el comportamiento de un huracán que puede ser explotada para obtener beneficios excesivos.

\subsection{Características de la negociación}

La unidad de negociación de las opciones HuRLO es una opción. Cotizan en dólares y centavos de dólar y su fluctuación mínima es de un céntimo de dólar $(0,01 \$)$. Si una persona posee más de 10.000 opciones en una serie y zona geográfica concretas debe proporcionar, a petición de Weather Risk Solutions o del mercado en el que se negocien, información sobre la naturaleza de la posición, la estrategia de negociación y la cobertura, si procede. A estos efectos, las opciones HuRLO que cubren la no llegada a tierra de huracanes (No Landfall HuRLO) se consideran una localización específica.

La negociación de las opciones HuRLO se produce cada año natural y comienza en invierno o a principios de primavera, antes de que exista el riesgo de ocurrencia de huracanes (la temporada oficial de huracanes en la costa Atlántica de EE.UU. va de junio a noviembre). Para cada una de las series creadas de la opción, la negociación termina el 15 de diciembre del año natural en que se negocian, cuando el huracán asociado a dicha serie toca tierra por primera vez (suponiendo que se cumple el umbral de daños establecido) o, si no se produce ningún huracán (o no se cumple el umbral de daños). La negociación en el mercado primario de las opciones HuRLO que cubren la no llegada a tierra de huracanes (No Landfall HuRLO) puede terminar antes, pero dicha negociación en el mercado secundario continua hasta que se produzca el evento, o hasta el 15 de diciembre, fecha a partir de la cual el riesgo de que se produzca un huracán en Estados Unidos se desvanece. Durante la temporada de huracanes, la actividad del mercado se suspende cuando el $\mathrm{CNH}$ emite una alerta de huracán para una o más zonas costeras, lo que indica el posible inicio de vientos huracanados con fuerza de tormenta tropical (o incluso más fuertes), es decir, vientos máximos sostenidos en superficie de 118,528 kilómetros/hora o superiores, en 36 horas o menos (Weather Risk Soluctions, 2010b).

Las especificaciones de las opciones HuRLO quedan predeterminadas el primer día de negociación de una serie concreta y todas ellas deben ajustarse a cualquier normativa gubernamental aplicable vigente en el momento del ejercicio. En este sentido, si un gobierno, agencia u organismo, con jurisdicción sobre la materia, emite una orden, resolución, directiva o ley incompatible con las especificaciones de los contratos, dicha orden, resolución, directiva o ley se interpreta como parte de estas especificaciones y todas las opciones HuRLO abiertas y las nuevas que se emiten quedan sujetas a ella.

Todas las operaciones con HuRLOs son compensadas y liquidadas a través de la Cámara de Compensación. Dicha liquidación es proporcional al número de opciones que posee cada participante en el mercado para el suceso cubierto (Weather Risk Soluctions, 2010c). 
El valor mínimo de liquidación es un porcentaje de la par, que establece Weather Risk Solutions antes de que se inicie la negociación de cada serie. De esta forma, si la par es de 1000 dólares y Weather Risk Solutions establece un valor mínimo de liquidación del $85 \%$ u 850 dólares, entonces el precio de las opciones se obtienen multiplicando 1.000 por las probabilidades de mercado y el titular de la opción nunca recibirá menos de 850 dólares si su HuRLO cubre el lugar donde efectivamente el huracán toca tierra por primera vez. Si eso sucede, el algoritmo de fijación de precios establecido aumenta el precio de ese resultado para garantizar que el valor de liquidación de esa opción no esté por debajo de los 850 dólares.

Las primas recaudadas por la compra de las opciones que cubren la llegada a tierra en primer lugar de un huracán en un determinado condado o región o aquellas que cubren la no llegada a tierra de ningún huracán durante un año natural, se invierten en un fondo común de riesgo mutualizado, de forma que, cuando se produce el resultado, el importe de este fondo total se reparte por igual entre los propietarios de la opción ganadora, independientemente del precio que hayan pagado por su opción. La liquidación es proporcional al número de opciones para el evento correcto que posee cada participante del mercado (Meyer et al. 2014).

\section{CÁlCUlo DEL PRECIO DE LAS OPCIONES HuRLO}

El precio de cada opción HuRLO para una serie determinada depende de un conjunto de factores basados en la interacción dinámica de las decisiones de negociación de todos los participantes en el mercado y de los datos históricos sobre el riesgo de huracanes que se incorporan a un algoritmo estocástico de control adaptativo en la plataforma de negociación que actualiza continuamente las probabilidades de mercado basándose en el comportamiento de compra de los participantes en el mercado en cada serie HuRLO

Siguiendo a Weather Risk Solutions (2010e), Wilks et al. (2014) y Boyer et al. (2020), para determinar el precio de las opciones HuRLO definimos, para una fecha y serie determinadas, las probabilidades de mercado, $\pi_{i}^{t}$, para cada resultado $i=0,1, \ldots, I$, siendo $I=75$ las zonas geográficas de primera llegada a tierra de un huracán de una serie determinada e $i=0$ la no llegada a tierra de ningún huracán. Estas probabilidades reflejan el comportamiento de compra de los participantes en el mercado en cada serie HuRLO y la suma de todas ellas debe ser igual a la unidad.

El precio de la opción para el resultado $i$ en un determinado momento $t$, que simbolizamos como $P_{i}^{t}$, se calcula de forma proporcional a estas probabilidades de mercado a partir de la siguiente expresión,

$$
P_{i}^{t}=\pi_{i}^{t} \cdot c \cdot e^{\frac{r \cdot j}{365}}
$$

donde $c$ es una constante que define la magnitud global de los precios y las liquidaciones en el mercado (esto es, el valor a la par establecido en 1.000 dólares), $r$ es el tipo de interés anual y $i$ es el número de días desde la apertura del mercado para el año en curso. 
Esta probabilidad, y los precios asociados a la misma, se recalcula después de la compra de cada opción HuRLO adicional. Esto es, si se adquiere un conjunto de 100 HuRLO para una región $i$, los precios se reajustan 100 veces a lo largo del proceso de realización del pedido de forma que cada una de las 100 opciones de este bloque costaría un poco más que la opción anterior comprado en el mismo bloque: la demanda de las opciones en la región $i$ provocaría un aumento en el precio de las opciones posteriores en esa región, y reduciría los precios de las opciones en los 74 resultados restantes de la serie concreta.

Los precios iniciales se asignan en función de las probabilidades históricas de riesgos climatológicos, $\pi_{i}^{0}$, de forma que el número inicial de opciones HuRLOs en cada resultado es igual, y viene dado por la siguiente expresión,

$\pi_{i}^{0}=\frac{M R P_{0}}{c}$

siendo $M R P_{0}$ la cuantía del fondo de riesgo mutualizado para cada serie al inicio de la negociación.

\section{CONCLUSIONES}

Las opciones HuRLO son derivados meteorológicos que posibilitan la cobertura de riesgos no incluidos en los contratos de seguros tradicionales como es el riesgo de huracán. Su objetivo no sustituir a los seguros de hogar, de interrupción de la actividad empresarial o de inundación sino complementar a los productos de seguros existentes. La estructuración de las opciones HuRLO en función de diferentes zonas geográficas costeras propensas a la ocurrencia de huracanes ofrece a los particulares y a las pequeñas y medianas empresas una posibilidad de cobertura anteriormente inexistente frente a este riesgo catastrófico además de posibilitar la especulación a aquellos inversores con mayores posibilidades de negocio. Además, la estructura del mercado en el que se negocian estos derivados permite que puedan realizarse compras sin necesidad de que exista una contrapartida y a un precio que varía a lo largo del tiempo de forma proporcional a la probabilidad de que el próximo huracán que toque tierra en EE.UU. se produzca en una zona concreta de la costa, según la percepción de los participantes en el mercado. Estos precios se obtienen a través de un algoritmo de control adaptativo y se recogen en un fondo común de riesgo mutualizado que sirve para indemnizar a los poseedores de los contratos que tienen el resultado ganador en función del número de contratos que posean.

\section{REFERENCIAS BIBLIOGRÁFICAS}

Boyer M., Breton, M., \& François, P. (2020). Designing Insurance against extreme weather risk: the case of HuRLOs. En Ecological, Societal, and Technological Risks and the financial sector. Edited by Thomas Walker-Dieter Gramlich-Mohammad BitarPedram Fardnia. Switzerland: Palgrave Macmillan, pp. 91-122 
Cummins, J. D., \& BARrieu, P. (2013). Innovations in insurance markets: Hybrid and securitized risk transfer solutions. In G. Dionne (Ed.), Handbook of insurance. Boston: Kluwer Academic Publishers.

Meyer, R. J., \& Horowitz, M. (2014). A novel financial market for mitigating hurricane risk. Part I: Market structure and model results. Weather, Climate, and Society, 6, 307-317.

Meyer, R. J., Horowitz, M., Wilks, D. S., \& Horowitz, K. A. (2014). A novel financial market for mitigating hurricane risk. Part II: Empirical validation. Weather, Climate, and Society, 6, 318-330.

Ou-YANG, C. (2010). Managing catastrophic risk by alternative risk transfer instruments. University of Pennsylvania Publicly accessible Dissertations 220. Recuperado de: http://repository.upenn.edu/edissertations/220.

Ou-YANG, C., \& DoherTy, N. (2011). Pari-mutuel insurance for hedging against catastrophic risk. Wharton School Working Paper \# 2011-08.

PÉRez-Fructuoso, M. J. (2005). La titulización del riesgo catastrófico: descripción y análisis de los cat bonds (Bonos de Catástrofes). Revista Española de Seguros, 121, 2005, pp. 75-92.

Weather Risk Solutions (2010a). Hurricane Risk Landfall Option-Questions \& Answers. Recuperado de: http://cyrus4.lightlink.com/wrs/faq.php

Weather Risk Solutions (2010b). Hurricane Risk Landfall Option - Contract Specifications. Recuperado de: http://cyrus4.lightlink.com/wrs/faq.php

Weather Risk Solutions (2010c). HuRLOS-Definitons \& Methodology. Recuperado de: http:// cyrus4.lightlink.com/wrs/faq.php

Weather Risk Solutions (2010d). WRS Electronic Trading Platform. Trading Rules. Recuperado de: http://cyrus4.lightlink.com/wrs/faq.php

Weather Risk Solutions (2010e). How Are HuRLOsTM Priced? Recuperado de: http://cyrus4. lightlink.com/wrs/faq.php

WILKS, D. S. (2010). A novel financial market structure for mitigating hurricane risk. Proceedings of the 20th Conference on Probability and Statistics in the Atmospheric Sciences. 